\title{
THE USE OF SHEARS TO CONSTRUCT PARADOXES IN $\mathbf{R}^{2}$
}

\author{
STANLEY WAGON
}

\begin{abstract}
It is shown that the addition of a certain shear transformation to the planar isometry group is sufficient to allow a Banach-Tarski type paradox to be constructed in $\mathbf{R}^{2}$. This paradox is then combined with a result of Rosenblatt to obtain a characterization of two-dimensional Lebesgue measure as a finitely additive measure.
\end{abstract}

1. Introduction. It is relatively easy to characterize $n$-dimensional Lebesgue measure as the only countably additive, translation invariant measure on the bounded Lebesgue measurable subsets of $\mathbf{R}^{n}$ that assigns measure one to (normalizes) the unit $n$-cube. But this characterization fails when finitely additive measures are considered. This was proved by Banach [1], in answer to a question of Ruziewicz: he showed (see [13]) that if $G$ is any Abelian (or solvable) group of isometries of $\mathbf{R}^{n}$ then there is a finitely additive, $G$-invariant measure on $\beta$ (the ring of bounded Lebesgue measurable subsets of $\mathbf{R}^{n}$ ) that normalizes the unit cube and is distinct from Lebesgue measure. Because this settles the uniqueness problem for translation invariant, finitely additive measures, Ruziewicz's problem has come to be interpreted as follows, where $G_{n}$ denotes the group of all isometries of $\mathbf{R}^{n}$, and $J^{n}=[0,1)^{n}$.

Ruziewicz's Problem. Is Lebesgue measure the only finitely additive $G_{n}$-invariant measure on $\beta$ that normalizes $J^{n}$ ?

The consideration of finite, rather than countable, additivity brings the uniqueness problem into the domain of functional analysis. In fact, Ruziewicz's Problem is equivalent to a question posed much earlier by Lebesgue [10, p. 99]: if an "integral", i.e., positive linear functional, on the space of bounded Lebesgue integrable functions on $\mathbf{R}^{n}$ is $G_{n}$-invariant and assigns value 1 to the characteristic function of $J^{n}$, does it necessarily satisfy the monotone convergence theorem? These problems are related because the monotone convergence property of an integral is equivalent to the countable additivity of the underlying measure.

Since $G_{1}$ and $G_{2}$ are solvable, Banach's work mentioned above settles Ruziewicz's Problem negatively in $\mathbf{R}^{1}$ and $\mathbf{R}^{2}$. For higher dimensions, however, no inroads were made on this problem for over 50 years. Then Rosenblatt [15] used techniques of functional analysis (invariant means) to solve the following variation of the problem. Let $\sigma$ denote the transformation of $\mathbf{R}^{n}$ given by $\sigma\left(x_{1}, x_{2}, \ldots, x_{n}\right)=$ $\left(x_{1}+x_{2}, x_{2}, \ldots, x_{n}\right)$.

THEOREM 1 (ROSENBLATt [15]). If $n \geq 2$ and $\mu$ is a finitely additive, $G_{n}$-invariant, $\sigma$-invariant measure on $\beta$ which normalizes $J^{n}$ and is absolutely continuous with respect to Lebesgue measure, then $\mu$ is Lebesgue measure.

Received by the editors September 1, 1981 .

1980 Mathematics Subject Classification. Primary 28D05, 03E20, 51M05.

Key words and phrases. Paradoxes, finitely additive measures. 
This theorem led Sullivan [19] and Margulis [11], independently, to obtain (using deep properties of the groups $G_{n}, n \geq 5$ ) a positive solution to Ruziewicz's Problem in $\mathbf{R}^{n}$ if $n \geq 5$. Moreover, Margulis [12] recently settled the remaining two cases, $\mathbf{R}^{3}$ and $\mathbf{R}^{4}$, positively. Thus Lebesgue measure is unique as a finitely additive, $G_{n}$-invariant measure iff $n \geq 3$.

Tarski had observed some time ago that if constructions similar to the BanachTarski Paradox exist with respect to a group $G$, then finitely additive, $G$-invariant measures are necessarily absolutely continuous with respect to Lebesgue measure. This is formalized and proved in $\S 3$ below. Because of the original Banach-Tarski Paradox using $G_{n}, n \geq 3$, Tarski's result means that the absolute continuity condition in Theorem 1 is unnecessary for $n \geq 3$. In this paper we show how a similar paradoxical construction can be given in the plane, using the group generated by $G_{2}$ and $\sigma$. When combined with the results of Tarski and Rosenblatt cited above, this yields a uniqueness theorem for 2-dimensional Lebesgue measure as a finitely additive measure.

The author acknowledges very helpful conversations and correspondence with Jan Mycielski and Joseph Rosenblatt.

2. A paradoxical decomposition of the square. First, some definitions.

DEFINITION. (a) Let a group $G$ act on a set $X$. Two subsets, $E_{1}, E_{2}$ of $X$ are $G$-equidecomposable if there are $g_{1}, \ldots, g_{n} \in G(n<\infty)$ and partitions $\left\{A_{1}, \ldots, A_{n}\right\},\left\{B_{1}, \ldots, B_{n}\right\}$ of $E_{1}, E_{2}$ respectively such that $g_{i}\left(A_{i}\right)=B_{i}$ for $i=$ $1, \ldots, n$.

(b) A nonempty subset $E$ of $X$ is $G$-paradoxical if $E$ has two disjoint subsets each of which is $G$-equidecomposable with $E$.

The celebrated Banach-Tarski Paradox [3] (see [15] for an elegant approach) states that the unit ball in $\mathbf{R}^{3}$ is $G_{3}$-paradoxical; more generally, they proved that if $n \geq 3$, then any two subsets of $\mathbf{R}^{n}$, each of which is bounded and has nonempty interior, are $G_{n}$-equidecomposable. No such paradoxes using isometries exist in $\mathbf{R}^{1}$ or $\mathbf{R}^{2}$. This follows from the theorem of Banach [1] that Lebesgue measure in $\mathbf{R}^{1}$ and $\mathbf{R}^{2}$ has an extension to a finitely additive, isometry invariant measure defined on all subsets of the line or plane. Von Neumann $[14$, p. 85] realized that paradoxes can be constructed in the plane, provided one enlarges the group of transformations that are used. More precisely, let $A_{2}$ be the group of all affine transformations of $\mathbf{R}^{2}$ that preserve area; $A_{2}$ consists of all maps $\tau \circ l$ where $\tau$ is a translation of the plane and $l$ is a linear transformation of $\mathbf{R}^{2}$ having determinant \pm 1 . Von Neumann proved that the unit square in $\mathbf{R}^{2}$ is $A_{2}$-paradoxical and consequently (for a reason explained below) any two bounded planar sets with nonempty interior are $A_{2}$-equidecomposable.

Von Neumann's proof is quite complicated. It is based on the use of small algebraically independent numbers to construct infinitely many non-Abelian free subgroups of $A_{2}$, whose generators are close to the identity (see [6, pp. 18-19]). In this section we give a simpler proof, which shows that the addition of only one affine transformation to $G_{2}$ suffices to get an action that is paradoxical. Let $\sigma_{1}$ be the shear of $\mathbf{R}^{2}$ given by $\left(\begin{array}{ll}1 & 2 \\ 0 & 1\end{array}\right)$, and let $G_{2}^{*}$ be the subgroup of $A_{2}$ generated by $G_{2}$ and $\sigma_{1}$.

THEOREM 2. The square, $J^{2}$, is $G_{2}^{*}$-paradoxical. In fact, any two bounded subsets of $\mathbf{R}^{2}$ with nonempty interior are $G_{2}^{*}$-equidecomposable. 
The presence of a free non-Abelian subgroup leads naturally to paradoxes because of the following proposition, which is implicit in [14], but really goes back to Hausdorff [7]. Note that the proof requires the Axiom of Choice.

PROPOSITION 1. If $F$, a free group of rank 2 , acts freely on $X$ (i.e., no $g \in F \backslash\{1\}$ has any fixed points), then $X$ is $F$-paradoxical.

ProOF. Suppose $F$ is freely generated by $\alpha_{1}, \alpha_{2}$. Then $F$ is paradoxical with respect to left multiplication,

$$
F \supseteq\left(W\left(\alpha_{1}\right) \cup W\left(\alpha_{1}^{-1}\right)\right) \cup\left(W\left(\alpha_{2}\right) \cup W\left(\alpha_{2}^{-1}\right)\right),
$$

where $W\left(\alpha_{i}^{ \pm 1}\right)$ is the set of words beginning with $\alpha_{i}^{ \pm 1}$, and

$$
F=W\left(\alpha_{i}\right) \cup \alpha_{i} W\left(\alpha_{i}^{-1}\right), \quad i=1,2 .
$$

If $M$ is a choice set for the $F$-orbits in $X$, then $X=\bigcup\{g(M): g \in F\}$ is a partition of $X$, and this partition allows the paradoxical decomposition of $F$ to be transferred to $X$.

It was known in the last century that $A_{2}$ has a free non-Abelian subgroup, because of Klein's theorem [8, pp. 218, 452] that $\mathrm{PSL}_{2}(\mathbf{Z}) \simeq \mathbf{Z}_{2} * \mathbf{Z}_{3}$ (see [9, p. 261]). But before this fact can be combined with Proposition 1 , two difficulties must be overcome: the free subgroup may not act on the square, and its action may have many fixed points.

Let $\sigma_{2}$ denote the shear represented by $\left(\begin{array}{ll}1 & 0 \\ 2 & 1\end{array}\right)$. Then it is a theorem of Sanov [16] (see also $[4,5]$ ), which is not hard to prove, that $\sigma_{1}, \sigma_{2}$ freely generate a free group of rank 2. Since $\sigma_{2}$ is in $G_{2}^{*}\left(\sigma_{2}=\rho^{-1} \sigma_{1}^{-1} \rho\right.$, where $\left.\rho=\left(\begin{array}{cc}0 & 1 \\ -1 & 0\end{array}\right)\right)$, this explicitly provides a free subgroup of $G_{2}^{*}$. Once the two problems mentioned in the previous paragraph are overcome, this free subgroup can be used, via Proposition 1, to prove Theorem 2.

The first problem is dealt with by reducing the action of the shears modulo 1 . Let $F$ be the group generated by $\sigma_{1}, \sigma_{2}$; as noted, $F$ is a free subgroup of $\mathrm{SL}_{2}(\mathbf{Z})$ of rank 2. Let $H$ be the subgroup of $G_{2}^{*}$ generated by $F$ together with the group $T$, of all translations of the plane.

Let $\sim$ denote the equivalence relation on $\mathbf{R}^{2}$ induced by the subgroup $\mathbf{Z}^{2}$ $\left(P \sim Q\right.$ iff $\left.P-Q \in \mathbf{Z}^{2}\right)$ and, for $P \in \mathbf{R}^{2}$, let $\hat{P}$ be the unique point in $J^{2}$ such that $\hat{P} \sim P$. For any $h \in H$, define $\hat{h}$, a function from $J^{2}$ to itself, by $\hat{h}(P)=\widehat{h(P)}$. If $h$ is $\sigma_{1}, \sigma_{2}$ or a translation, then $\hat{h}$ is an area preserving bijection of $J^{2}$ onto itself. Let $\hat{H}=\{\hat{h}: h \in H\}$ and define $\hat{F}, \hat{T}$ similarly. It follows from the next proposition that $\hat{H}, \hat{F}$ and $\hat{T}$ are all groups that act on $J^{2}$.

PROPOSITION 2. If $h_{1}, h_{2} \in H$, then $\widehat{h_{1} h_{2}}=\hat{h}_{1} \hat{h}_{2}$.

Proof. By induction on the length of $h$ expressed in terms of $\sigma_{1}, \sigma_{2}$ and translations, it is routine to show that if $P \sim Q$ then $h(P) \sim h(Q)$; this uses the fact that $\sigma_{i} \in \mathrm{SL}_{2}(\mathbf{Z})$ and hence $\sigma_{i}\left(\mathbf{Z}^{2}\right) \subseteq \mathbf{Z}^{2}$. Now, $\hat{h}_{1} \hat{h}_{2}(P)=\hat{h}_{1} \widehat{h_{2}(P)}=\widehat{h_{1}\left(h_{2}(P)\right)}=\widehat{h_{1}\left(h_{2}(P)\right)}=\widehat{h_{1} h_{2}}(P) \quad\left(\right.$ since $\left.h_{2}(P) \sim \widehat{h_{2}(P)}\right)$.

Proposition 3. The mapping $h \mapsto \hat{h}$ is an isomorphism of $F$ with $\hat{F}$. Therefore $F$ is a free group on the two generators $\hat{\sigma}_{1}, \hat{\sigma}_{2}$. 
ProOF. It suffices to show that the kernel of the mapping is trivial since, by Proposition 2, the mapping is a homomorphism and, clearly, it is surjective. Suppose $\hat{h}=1$. Since $\hat{h}$ is the composition of $h$ with a piecewise translation, there is some $\tau \in T$ and an open set $U$ (the interior of a polygon) such that $1=\hat{h}=\tau h$ on $U$. But $h$ and $\tau^{-1}$ are affine maps which, if they agree on 3 noncollinear points, agree everywhere. Therefore $h=\tau^{-1}$ which, since $h$ is a linear transformation, is impossible unless $\tau=1$, as required.

These two propositions show how certain actions can be modified to act on the square. Still, the transformations of the square in $\hat{F}$ have many fixed points, and the rest of the proof of Theorem 2 consists of using the translations to, in an appropriate sense, eliminate them.

ProOF OF THEOREM 2. Since $H \subseteq G_{2}^{*}$ it suffices to show that $J^{2}$ is $H$ paradoxical. If we simply ignore the troublesome fixed points of the action of $F$ on $J^{2}$ there is no problem. More precisely, let $D=\left\{P \in J^{2}: \hat{h}(P)=P\right.$ for some $\hat{h} \in \hat{F} \backslash\{1\}\}$. Then $\hat{F}$ acts freely on $J^{2} \backslash D$ whence, by Propositions 1 and $3, J^{2} \backslash D$ is $\hat{F}$-paradoxical. This implies that $J^{2} \backslash D$ is $H$-paradoxical because every transformation in $\hat{F}$ is, piecewise, in $H$ (since $T \subseteq H$ ). Paradoxical decompositions are invariant under equidecomposability, whence the proof will be complete once we show that $J^{2} \backslash D$ is $T$-equidecomposable, and therefore $H$-equidecomposable, with $J^{2}$.

Since every point in $D$ is a fixed point of an affine map, and since $\hat{F}$ is countable, $D$ may be split into $D_{0} \cup D_{1}$ where $D_{0}$ is a countable set of points and $D_{1}$ is a countable set of line segments. We shall exploit ideas of Sierpiński [17] and Hausdorff [7] to show that $J^{2}$ and $J^{2} \backslash D$ are $T$-equidecomposable. They considered the case where the set of fixed points is countable, but the set $D_{1}$ bears enough resemblance to a countable set to allow the use of their techniques.

We claim that there is some translation $\tau$ such that $D \cap \hat{\tau}^{n}(D)$ is countable for any integer $n \neq 0$. Enumerate the segments in $D_{1}$ as $S_{0}, S_{1}, S_{2}, \ldots$ Suppose $n$, as well as $i, j$ with $0 \leq i, j$ and $i \neq j$ are given integers. If $S_{i}$ and $S_{j}$ are not parallel we do nothing, for then, no matter what $\tau$ is, $\hat{\tau}^{n}\left(S_{j}\right)$ intersects $S_{i}$ in at most a single point. But if they are parallel, choose a point $P$ on $S_{j}$ and discard all translations $\tau$ such that, for some $\left(m_{1}, m_{2}\right) \in \mathbf{Z}^{2}, \tau^{n}(P)$ lies on the doubly infinite line containing the segment $S_{i}+\left(m_{1}, m_{2}\right)$. This guarantees that if $\tau$ is not discarded, then $\hat{\tau}^{n}\left(S_{j}\right) \cap S_{i}=\emptyset$. For each of the countably many triples $n, i, j$, at most countably many lines of translations (where $T$ is identified with $\mathbf{R}^{2}$ ) are discarded, and hence altogether only countably many lines of translations are discarded. Therefore on any nondiscarded line only countably many translations are discarded, and so there must be one, call it $\tau$, left over. Now, $\tau$ is as desired; for if $D \cap \hat{\tau}^{n}(D)$ is uncountable, then some segment in $D_{1}$ must overlap with one in $\hat{\tau}^{n}\left(D_{1}\right)$, and any translation for which this could happen was discarded.

Let $P=\bigcup\left\{D \cap \hat{\tau}^{n}(D): n= \pm 1, \pm 2, \ldots\right\} ; P$ is a countable subset of $D$. We claim that $J^{2} \backslash P$ is $\hat{T}$-equidecomposable with $J^{2} \backslash D$. It follows from the definition of $P$ that the sets $\hat{\tau}^{n}(D \backslash P), n \geq 0$, are pairwise disjoint, and that each is disjoint from $P$. Hence the situation is as illustrated in the figure below. Let $A=\bigcup_{n=0}^{\infty} \hat{\tau}^{n}(D \backslash P)$. Then $J^{2} \backslash P$ splits into $A$ and $\left(J^{2} \backslash P\right) \backslash A$. Since

$$
\hat{\tau}(A)=\bigcup_{n=1}^{\infty} \hat{\tau}^{n}(D-P)=\left(J^{2} \backslash D\right) \backslash\left(\left(J^{2} \backslash P\right) \backslash A\right),
$$


this shows that $J^{2} \backslash P$ is equidecomposable with $J^{2} \backslash D$ via $\hat{\tau}$ and the identity, as claimed.

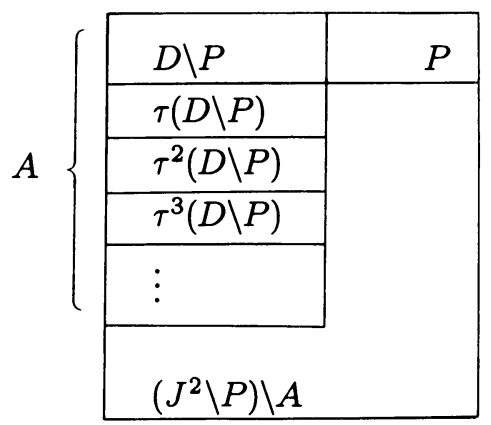

A similar argument, though much simpler, shows that for any countable subset $C$ of $J^{2}, J^{2}$ and $J^{2} \backslash C$ are $\hat{T}$-equidecomposable. Therefore $J^{2}$ is $\hat{T}$-equidecomposable with $J^{2} \backslash P$ and hence with $J^{2} \backslash D$. As discussed at the beginning of the proof, this implies that $J^{2}$ is $T$-equidecomposable with $J^{2} \backslash D$; this completes the proof of the first assertion of the theorem.

To obtain the rest of the theorem we must appeal to a Schröder-Bernstein type of theorem for equidecomposability (see $[2 ; 3$, Théorème 8$]$ or $[11$, p. 81]; the proof is essentially identical to the classical case for cardinality): if $A$ is $G$-equidecomposable with a subset of $B$ and $B$ is $G$-equidecomposable with a subset of $A$, then $A$ and $B$ are $G$-equidecomposable with each other. Now, suppose $K$ and $L$ are two given bounded subsets of the plane with nonempty interior. Then $L$ contains a square (with interior) $S$ and, because the unit square is $G_{2}^{*}$-paradoxical, so is $S$. Repeated duplication of $S$ yields that $S$ is $G_{2}^{*}$-equidecomposable with a square containing the bounded set $K$. Thus $K$ is $G_{2}^{*}$-equidecomposable with a subset of $S$ and hence with a subset of $L$. We may repeat this argument, interchanging the roles of $K$ and $L$ so that the Schröder-Bernstein Theorem may be applied to get the $G_{2}^{*}$-equidecomposability of $K$ and $L$.

3. An application of the paradox. Tarski's observation that the Banach-Tarski Paradox can be used to eliminate the absolute continuity condition in Theorem 1 if $n \geq 3$ may be formalized as follows.

PROPOSITION 4. If $G$ is a group of Lebesgue measure preserving transformations of $\mathbf{R}^{n}$ that contains all translations and that is rich enough so that $J^{n}$ is $G$ paradoxical, and if $\mu$ is a finitely additive, $G$-invariant measure on $\beta$ that normalizes $J^{n}$, then $\mu$ vanishes on the sets in $\beta$ having Lebesgue measure zero.

Proof. Suppose $E$ is a bounded set having Lebesgue measure zero and $I$ is a cube large enough to contain $E$. Since $J^{n}$ is $G$-paradoxical it follows (see the end of the proof of Theorem 2) that any two cubes are $G$-equidecomposable. Hence, for any $\epsilon>0, I$ is $G$-equidecomposable with a cube of volume $\epsilon$. Now, $\mu$ agrees with volume on cubes (one first uses translation invariance to show that $\mu$ is correct on cubes of side-length $\frac{1}{2}, \frac{1}{4}, \frac{1}{8}, \ldots$ and then uses such cubes to approximate the others), but this does not mean that $\mu(I)=\epsilon$. For the pieces involved in the $G$-packing of $I$ into the $\epsilon$-cube may not be Lebesgue measurable, and hence not $\mu$-measurable. But arbitrary subsets of Lebesgue measure zero sets are always Lebesgue measurable, whence the intersections of the pieces with $E$ are in $\beta$, and 
therefore are $\mu$-measurable. Thus, by the finite additivity and $G$-invariance of $\mu$, the $G$-equidecomposability of $I$ with an $\epsilon$-cube implies that $\mu(E) \leq \epsilon$. But $\epsilon$ was arbitrary, so $\mu(E)=0$.

Now, Theorem 2 implies that $J^{2}$ is paradoxical with respect to the group generated by $G_{2}$ and $\sigma$; this is because $\sigma_{1}=\sigma^{2}$ and so $G_{2}^{*}$ is contained in the group generated by $G_{2}$ and $\sigma$. This allows Tarski's proposition to be combined with Rosenblatt's Theorem 1 to obtain the following characterization of Lebesgue measure in the plane.

THEOREM 3. Lebesgue measure is the only finitely additive, $G_{2}$-invariant, $\sigma$ invariant measure on $\beta$ that normalizes the unit square.

This should be contrasted with Banach's result that this characterization fails without the hypothesis of $\sigma$-invariance.

4. Tarski's circle squaring problem. The existence of finitely additive, isometry invariant extensions of Lebesgue measure to all subsets of the plane (Banach; see the beginning of $\S 2$ ) implies that $G_{2}$-equidecomposable Lebesgue measurable sets must have the same Lebesgue measure. This led Tarski [20] to ask whether a circle (with interior) is $G_{2}$-equidecomposable with a square of the same area. There are some partial results on this question (see [21] for a survey) but they do not provide much evidence as to which answer to Tarski's quesiton is more plausible.

It follows from Theorem 2 that the addition of a shear transformation allows the decomposition of a circle into a square to be carried out. More precisely, a circle is $G_{2}^{*}$-equidecomposable with a square. Unfortunately, allowing transformations by the shear $\sigma_{1}$ changes the problem so dramatically (since, by Theorem 2, a circle is $G_{2}^{*}$-equidecomposable with any square, no matter how large or small) that this result does not really indicate that a positive answer to Tarski's original question is more likely than a negative one.

\section{REFERENCES}

1. S. Banach, Sur le problème de la mésure, Fund. Math. 4 (1923), 7-33.

2. _ Un théorème sur les transformations biunivoques, Fund. Math. 6 (1924), 236-239.

3. S. Banach and A. Tarski, Sur la décomposition des ensembles de points en parties respectivement congruentes, Fund. Math. 6 (1924), 244-277.

4. J. L. Brenner, Quelques groupes libre de matrices, C. R. Acad. Sci. Paris 241 (1955), 1689-1691.

5. K. Goldberg and M. Newman, Pairs of matrices of order two which generate free groups, Illinois J. Math. 1 (1957), 446-448.

6. F. P. Greenleaf, Invariant means on topological groups, Van Nostrand, New York, 1969.

7. F. Hausdorff, Bemerkung über den Inhalt von Punktmengen, Math. Ann. 75 (1914), 428-433.

8. F. Klein and R. Fricke, Theorie der elliptischen Modulfunktionen, vol. 1, Teubner, Leipzig, 1890.

9. A. G. Kurosh, The theory of groups, vol. II, Chelsea, New York, 1956.

10. H. Lebesgue, Leçons sur l'intégration, Gauthier-Villars, Paris, 1904 (2nd ed., 1928; reprinted Chelsea, New York, 1973).

11. G. A. Margulis, Some remarks on invariant means, Monatsh. Math. 90 (1980), 233-235.

12. , Finitely additive invariant measures on Euclidean spaces, Preprint, 1981.

13. J. Mycielski, Finitely additive measures. I, Colloq. Math. 42 (1979), 309-318.

14. J. von Neumann, Zur allgemeinen Theorie des Masses, Fund. Math. 13 (1929), 73-116.

15. J. Rosenblatt, Uniqueness of invariant means for measure-preserving transformations, Trans. Amer. Math. Soc. 265 (1981), 623-636.

16. I. N. Sanov, A property of a representation of a free group, Dokl. Akad. Nauk SSSR 57 (1947), 657-659. (Russian) 
17. W. Sierpinski, Sur l'équivalence des ensembles par décomposition en deux parties, Fund. Math. 35 (1948), 151-158.

18. K. Stromberg, The Banach-Tarski paradox, Amer. Math. Monthly 86 (1979), 151-161.

19. D. Sullivan, For $n>3$ there is only one finitely additive rotationally invariant measure on the $n$-sphere defined on all Lebesgue measurable subsets, Bull. Amer. Math. Soc. (N.S.) 4 (1981), 121-123.

20. A. Tarski, Probleme 38, Fund. Math. 7 (1925), 381.

21. S. Wagon, Circle-squaring in the twentieth century, Math. Intelligencer 3 (1981), 176-181.

Department of Mathematics, Smith College, Northampton, MassachuSETTS 01063 